\title{
Um panorama das pesquisas e publicações sobre mudança organizacional no Brasil
} An overview of Brazilian research and publications
about organizational change
Un panorama de las investigaciones y publicaciones
sobre el cambio organizacional en el Brasil

\author{
Elaine Rabelo NEIVA ${ }^{1}$ \\ Maria das Graças Torres da PAZ \\ Universidade de Brasília, Brasília, DF, Brasil
}

ResumO Este trabalho analisa pesquisas brasileiras sobre mudança organizacional divulgadas em periódicos científicos de administração, sociologia, ciências da saúde e psicologia entre os anos 2000 e 2010, avaliados com classificação A ou B no Qualis/CAPES. Os artigos selecionados, por meio de título e resumos, foram classificados por juízes, que os liam e faziam registros sobre eles, com base em um sistema padronizado, previamente desenvolvido, de análise e categorização de investigações empíricas. A maioria dos textos apresenta um objeto de estudo relacionado à mudança organizacional e aborda processos de mudança contínua e seu conteúdo. É possível concluir que o objeto de estudo mudança organizacional foi classificado em termos de escopo e intensidade maiores, mas com caráter de continuidade no tempo. Quanto à abordagem metodológica, os resultados mostram estudos que adotam prioritariamente a abordagem metodológica de estudos de caso qualitativos, que utilizam a análise de conteúdo como único método de análise de dados. Assim, conclui-se que há uma tendência a valorizar a abordagem metodológica qualitativa e a escassez de fundamentação teórica. A partir das lacunas encontradas, o artigo sugere diversificar as abordagens metodológicas e apurar a fundamentação teórica dos estudos sobre o tema.

Palavras-chave:

Mudança organizacional; avaliações das mudanças organizacionais; revisão bibliográfica; comportamento organizacional.

Abstract This paper analyzes Brazilian research on organizational change published in scientific journals on administration, sociology, psychology and health sciences, between 2000 and 2010, evaluated with a rating of $\mathrm{A}$ or $\mathrm{B}$ in the Qualis/CAPES. The articles selected, by means of title and abstracts, were classified by judges, who read them and created records on them, based on a previously developed standardized system of analysis and categorization of empirical investigations. Most of the texts present an object of study related to organizational change and address ongoing change processes and their content. It was concluded that the study subject, organizational change, was classified in terms of a broader scope and intensity, but with a character of continuity over time. Regarding the methodological approach, the results show studies that primarily adopt the methodological approach of qualitative case studies, using content analysis as the sole method of data analysis. Thus, it is concluded that there is a tendency to value the qualitative methodological approach and toward the lack of a theoretical basis. From the gaps found, the article suggests diversifying the methodological approaches and determining the theoretical basis of studies on the subject.

Keywords:

Organizational change; assessments of organizational change; bibliographic review; organizational behavior. 
Este trabajo analiza investigaciones brasileñas acerca del cambio organizacional con base en los artículos publicados en revistas científicas de administración, sociología, ciencias de la salud y psicología cuya evaluación ha sido de A o B de acuerdo a la clasificación Qualis/ CAPES. El período inestigado fue del 2000 al 2010. Los artículos, seleccionados por su título y resúmenes, fueron clasificados por jueces, que leían los artículos y hacían anotaciones sobre ellos, con base en un sistema estandarizado, desarrollado previamente, de análisis y categorización de las investigaciones empíricas. La mayoría de los textos presenta que el objeto de estudio se centró en el cambio organizacional, que las investigaciones abordan los procesos continuos de cambio continuo y que ellas describen los contenidos del cambio. En cuanto al enfoque metodológico, los resultados muestran que los estudios adoptan principalmente el enfoque metodológico de estudios de caso, cualitativos, utilizando el análisis de contenido como el único método de análisis de datos. Se concluye que existe una tendencia a valorar el enfoque metodológico cualitativo y que los estudios no muestran un marco teórico consistente. A partir de las carencias que se encuentran en las publicaciones, el artículo sugiere diversificar los enfoques metodológicos y construir la base teórica de los estudios sobre el tema.

Palabras-clave:

Cambio organizacional; las evaluaciones de los cambios organizacionales; revisión de la literatura; el comportamiento organizacional.

A

rmenakis e Bedeian (1999), em uma clássica revisão de literatura sobre mudança organizacional dividem as pesquisas sobre o tema nas seguintes categorias: conteúdo, contexto, processo e critério. Em conteúdo, estão os trabalhos sobre diagnóstico para mudança que abordam o que está sendo alvo das intervenções para alcançar esse objetivo. O tema contexto envolve pesquisas sobre as forças existentes nos ambientes interno e externo à organização que influenciam o processo de mudança organizacional. Tais pesquisas contemplam temas como desregulamentação, inércia organizacional, mudança ambiental e reorientação organizacional, inadaptação das organizações mais velhas ao mercado, tamanho organizacional e resposta a mudança, entre outros.

A categoria processo envolve as ações realizadas nas fases de implantação da mudança e as subsequentes, que descrevem como os indivíduos a experimentam. Por fim, os autores propõem o tema critério que abarca outros fatores para mensuração dos resultados dos esforços de mudança organizacional, apontando critérios comportamentais e afetivos aliados aos de produtividade. Apesar de o referido artigo não apresentar uma definição de mudança organizacional, os autores retratam a literatura sobre o tema na tentativa de explicitar os avanços alcançados até aquele momento no campo de estudos, e de fornecer uma indicação acurada das principais perspectivas dominantes no pensamento contemporâneo desse construto (Armenakis \& Bedeian, 1999).

Considerando a necessidade explicitada pelos autores acima citados em compreender a evolução do pensamento no tema de mudança organizacional (MO), este artigo tem por objetivo realizar uma revisão de literatura sobre $\mathrm{MO}$ em estudos brasileiros entre 2000 e 2010. Para tanto, as literaturas nacional e internacional foram utilizadas para a elaboração de definições e categorias, descritas na Tabela 3, e, a partir destas, foram analisados artigos de periódicos das áreas de psicologia, administração, sociologia, antropologia e ciências da saúde, presentes em bases de dados nacionais.

\section{O conceito de mudança organizacional na literatura}

Mudança organizacional é um fenômeno multifacetado que ocupa lugar de destaque nos estudos realizados na área de comportamento organizacional (seja ele macro, meso ou micro). $\mathrm{O}$ interesse por esse fenômeno data dos anos de 1970/1980, nos quais estudiosos focavam as técnicas de desenvolvimento organizacional e buscavam provocar mudanças nas organizações de trabalho por meio de alterações nos comportamentos individuais (Neiva, 2004). Mesmo com estudos iniciados há mais de 20 anos, a MO é de difícil conceituação, e a literatura apresenta uma miríade de conceitos e definições.

A definição de $\mathrm{MO}$ permanece tão problemática quanto outros grandes construtos da área de organizações e trabalho (p. ex., cultura e clima organizacionais, estratégia). Lima e Bressan (2003) realizaram um levantamento na literatura sobre o tema em um intervalo de aproximadamente 20 anos e identificaram a falta de um eixo norteador comum a todas as definições. Contudo, também identificaram dimensões congruentes da mudança: (a) intencionalidade, (b) relevância do impacto da mudança, (c) construção social da mudança, (d) temporalidade e (e) reposta ao ambiente. 
Outro aspecto relevante sobre a MO no levantamento das autoras é a evolução das definições ao longo dessas duas décadas. Observa-se que os conceitos apresentados partem do ponto de vista de que a mudança é planejada e apoiada pela administração da organização, passando por uma conotação mais abstrata que a considera uma observação empírica individual, chegando a uma conceituação mais ampla que engloba toda a alteração planejada, ou não, com impactos na organização.

É importante ressaltar que há, também, duas tendências claras nas definições apresentadas. Ou são enfatizadas intervenções e seus impactos, ou é enfatizada a construção social da mudança por meio da percepção e da atribuição de significados. Alguns autores atribuem essa diferenciação à concepção epistemológica do fenômeno (Van de Ven \& Poole, 1995, 2005), questão que será retomada mais adiante.

Segundo Neiva (2004), para a análise do conceito, por meio do uso da linguagem cotidiana (Harzem \& Miles, 1978), o uso mais típico da palavra mudança parece ser o que indica alteração, modificação apresentada por um objeto, pessoa ou situação vista sob períodos temporais diferentes. Há a comparação de um objeto, uma pessoa ou uma situação em pelo menos duas ocasiões e a identificação de diferenças ou alterações em suas características ou atributos. Seu uso é bastante vago, podendo ser aplicado a inúmeros objetos ou situações, e o tempo é delimitador de seu uso. O conceito de mudança é relativo/relacional, pois são descritas comparações entre um objeto ou contexto em situações diferentes no decorrer de determinado período de tempo. Não faz sentido perguntar o que é a mudança em si, porque não diz respeito a um objeto específico. Um conceito relativo não é usado para apenas um episódio.

Ainda segundo essa autora, mudanças dizem respeito a avaliações feitas por indivíduos para estabelecer comparações entre objetos, eventos ou contextos. Na linguagem cotidiana, quando fazemos tais comparações e indicamos que houve mudança, as pessoas logo perguntam: "Que tipo de mudança?". É necessário complementar seu sentido, caracterizando-a em termos de quais atributos mudaram e com qual intensidade.

Lima e Bressan (2003) apontaram algumas dimensões que podem ser consideradas na definição de MO e na apresentação de tipologias que abordam a amplitude do fenômeno. Entre os aspectos, podem ser citados: (a) continuidade versus descontinuidade no tempo, (b) objeto da mudança (organização como um todo ou apenas subsistemas organizacionais), (c) intensidade da mudança (intensa ou gradual), (d) tempo da resposta a eventos externos (antecipação ou reação), (e) velocidade da mudança (devagar ou rápida), (f) intencionalidade versus emergência da mudança, (g) atores que exercem papel fundamental na mudança (alta direção versus membros da organização), (h) papel do agente de mudança e (i) prazo em que a mudança ocorre. Na literatura, há certa preocupação tanto em definir quanto em classificar e/ou tipificar a mudança como mecanismo para o estudo e a compreensão do fenômeno. Na próxima seção, serão apresentadas algumas classificações encontradas na literatura que auxiliarão na apreensão mais ampla do fenômeno aqui apresentado.

\section{Tipologia sobre mudança organizacional}

Vários autores, em momentos diferenciados e seguindo orientações diversas, apresentaram tipologias, muitas vezes desvinculadas de conceitos, sobre a MO. Weick e Quinn (1999) sugeriram um importante contraste nas pesquisas sobre mudanças: a distinção entre mudança episódica, descontínua e intermitente e mudança contínua, incremental e constante. Segundo os autores, essa distinção é central em sua conceituação, conforme mostra a Tabela 1, em que são apresentadas as características básicas das mudanças episódicas e contínuas, as dimensões da concepção de organização, o arcabouço analítico e as proposições sobre a intervenção dela.

TABELA 1. Tipologia da mudança

\begin{tabular}{|c|c|c|}
\hline & Mudança episódica & Mudança contínua \\
\hline Concepção de organização & $\begin{array}{l}\text { As organizações são estáveis e funcio- } \\
\text { nam com padrões automatizados em que } \\
\text { prepondera a inércia. }\end{array}$ & $\begin{array}{l}\text { Organizações são emergentes e auto-organi- } \\
\text { záveis. }\end{array}$ \\
\hline Características da mudança & $\begin{array}{l}\text { São pouco frequentes, descontínuas e } \\
\text { intencionais. }\end{array}$ & São constantes e cumulativas. \\
\hline Arcabouço analítico & $\begin{array}{l}\text { Mudança equivalente à interrupção oca- } \\
\text { sional do equilíbrio, dramática e controla- } \\
\text { da externamente. Constitui uma falha da } \\
\text { organização em se adaptar a um ambien- } \\
\text { te em mudança. }\end{array}$ & $\begin{array}{l}\text { Mudança equivalente a um padrão de alteração } \\
\text { contínua em processos de trabalho e práticas } \\
\text { sociais. Ela é cumulativa e motivada por instabi- } \\
\text { lidade organizacional e por reações cotidianas. }\end{array}$ \\
\hline
\end{tabular}

Nota. Adaptado de Weick e Quinn (1999). 
Os autores ressaltam, ainda, o questionamento sobre o desenvolvimento da mudança organizacional como um corpo de conhecimento refutável e cumulativo. Macy e Izumi (1993, citado por Weick \& Quinn, 1999) sobre o trabalho de Kahn (1974), fazem a seguinte afirmativa de que, no campo da MO "poucas proposições teóricas são repetidas sem dados adicionais ou desenvolvimento, alguns conselhos são reiterados sem provas ou evidências e poucos estudos e observações empíricas são apresentados com reverência, sem, contudo, serem refinados ou explicados" (p. 363).

A indicação do escopo das alterações realizadas na organização é um fator extremamente importante para a especificação da tipologia de mudanças. Para Burke e Litwin (1992), a mudança transformacional é consequência da interação e das pressões do ambiente externo, demandando novos comportamentos dos empregados. Algumas características organizacionais possuem grande peso (nível de influência) no processo de mudança, pois, quando sofrem alterações, tendem a alterar os outros. Mudanças na missão, na estratégia, na liderança ou na cultura possuem mais peso do que fatores como estrutura, práticas gerenciais e sistemas. Portanto, segundo Burke e Litwin (1992) e Burke (2008), as organizações realizam mudanças transformacionais quando alteram ambiente externo, missão, estratégia, liderança e cultura organizacional. Já as mudanças transacionais alteram aspectos como estrutura, práticas gerenciais e sistemas de informação e de procedimentos nas organizações.

Conforme levantado por Weick e Quinn (1999), muitos autores consideram que a questão da conceituação e das tipologias de mudança é fruto de concepções teóricas e visões de organização diferenciadas. O campo dos estudos organizacionais é resultado de verdadeiras polêmicas em relação ao conteúdo de alguns referenciais teóricos, e um deles é o tema mudança (Pettigrew, Woodman, \& Cameron, 2001).

Van de Ven e Poole (2005) asseguram que os estudiosos possuem diferentes visões sobre organizações que oscilam entre considerá-las entidades ou processos, e entre usar métodos de variância ou de processo para conduzir pesquisas. Os autores argumentam que duas definições de mudança são usadas nos estudos organizacionais: (a) uma diferença observada ao longo do tempo em uma entidade organizacional ou em dimensões selecionadas e (b) uma narrativa descrevendo uma sequência de eventos sobre como o desenvolvimento e as mudanças ocorrem.

Quando o primeiro conceito é usado, a mudança é definida pela Teoria Metodológica da Variância, em que é representada por uma variável dependente, cuja variabilidade é estatisticamente explicada por um conjunto de variáveis independentes. A segunda definição trata a mudança a partir da Teoria de Processo, que a concebe como sequência e ordenação temporal de eventos, em que os de mudança ocorrem baseados em uma história ou narrativa histórica (Van de Ven \& Poole, 2005).

Sem desconsiderar a discussão sobre as perspectivas teóricas e metodológicas que fundamentam tais estudos, este trabalho pretende descrever e analisar a produção científica nacional sobre MO durante o período de 2000 a 2010. Tal circunscrição de período se deve à necessidade de analisar uma década de produção. A partir da proposta de análise para revisões bibliográficas elaboradas por Borges-Andrade, Coelho Jr. e Queiroga (2006), esta revisão acrescentou outras categorias sobre MO, construídas a partir das revisões sistemáticas de literatura internacional sobre o tema (Armenakis \& Bedeian, 1999; Pettigrew et al., 2001; Weick \& Quin, 1999).

\section{MÉTODO}

Para realizar a revisão de literatura, foi adotado o seguinte procedimento para a seleção dos artigos: algumas bases de dados foram consultadas para identificar quais artigos, publicados durante a primeira década deste século em periódicos brasileiros, referiam-se à temática de MO. Os periódicos escolhidos para integrar a base de dados deveriam ser indexados e conter avaliações pela CAPES, nos níveis A e B, conforme a Tabela 2.

O período consultado se justifica em virtude de os artigos publicados sobre o tema a partir de 1999 sugerirem avanços, abarcando a última década de publicações. Foram consultadas as bases de dados Google Acadêmico Nacional, SciELO, PePSIC e os sites dos periódicos. Todos os artigos indicados pela base foram revistos para verificar se realmente abordavam o tema mudança organizacional. Em um primeiro momento, consultando o resumo e o título, foram selecionados 110 artigos. Dessa primeira etapa participaram nove pessoas. 
TABELA 2. Descrição dos periódicos nacionais analisados

Periódicos consultados nas disciplinas de administração, ciências da saúde, psicologia e sociologia

Cadernos de Saúde Pública

Ciência \& Saúde Coletiva

Comportamento Organizacional e Gestão

Estudos de Psicologia - PUC Campinas

Estudos de Psicologia (UFRN)

Organizações e Sociedade - O\&S (UFBA)

Psicologia, Ciência e Profissão (CFP)

Psicologia em Estudo (UEM)

Psicologia: Organizações e Trabalho - rPOT (SBPOT)

Psicologia: Reflexão e Crítica (UFRS)

Psicologia: Teoria e Pesquisa (UnB)

Psico PUC RS

Psico USF

Revista de Administração Contemporânea - RAC (ANPAD)

Revista de Administração de Empresas - RAE (FGV-SP)

Revista de Administração do Mackenzie (UPM)

Revista de Administração Pública - RAP (FGV-RJ)

Revista de Administração da USP - RAUSP

Revista Eletrônica de Administração - ReAd (URGS)

Revista Gestão Industrial

Revista Gestão \& Produção

Revista: Sociologia, Problemas e Práticas

Total

22 periódicos

Em paralelo ao levantamento dos artigos, o mesmo grupo citado elaborou categorias para avaliação e classificação deles. Em seguida, foi feita uma distribuição destes entre os nove participantes, ficando cada um, em média, com 10 artigos para analisar. Nessa segunda fase, cada pessoa ficou responsável por analisar o artigo na íntegra e, a partir disso, classificá-lo segundo as categorias descritas na Tabela 3.

A presente categorização foi elaborada a partir da literatura sobre $\mathrm{MO}$ e categorias presentes em uma base de dados elaborada por Borges-Andrade, Coelho Jr. e Queiroga (2006) com complementação metodológica de Rossi et al. (1999). Em um terceiro momento, os dados foram revisados por três pesquisadores. Nessa revisão, além da padronização e da mudança de alguns dados, 13 artigos foram descartados por se tratarem de dados duplicados, resultando em 97 artigos $(n=97)$.

TABELA 3. Descrição e definição das categorias usadas para análise dos artigos

\section{Categoria}

Descrição da categoria de análise

Área de conhecimento (p. ex., administração, Avalia a área de conhecimento dos autores do artigo.

psicologia)

Desenho de investigação (survey, experimental No caso de estudos empíricos, avalia se o desenho foi um survey, se houve tentativa ou pesquisa-ação) de desenho pré-experimental ou se houve um desenho experimental ou quaseexperimental.

Finalidade do estudo

Avalia se o estudo pretendeu gerar conhecimento, gerar instrumento para outras pesquisas ou gerar tecnologia.

Foco no processo, nos resultados (critério), no Considerando as definições de Armenakis e Bedeian (1999), classifica o artigo em um conteúdo ou no contexto dos focos apresentados.

Mensuração das mudanças ou utilização de Avalia se a percepção de mudança foi investigada por parte dos sujeitos respondentes medidas perceptuais nos artigos e, caso a resposta seja positiva, se houve percepção de mudança (Rossi, Freeman, \& Lipsey, 1999; Woodman, 1989).

Mudança contínua ou episódica/descontínua

Avalia se a mudança retratada pelo artigo foi gradual, lenta ou ocorrida como um episódio rápido e pontual, que abala a organização (Burke, 2008; Cummings \& Worley, 2005; Van de Ven \& Poole, 2005; Weick \& Quinn, 1999).*

Mudança organizacional ou mudança nas Avalia o escopo da intervenção analisada pelo artigo no caso de artigo empírico. A organizações intervenção envolveu várias áreas ou apenas algumas unidades da organização. Também foi avaliado se as intervenções focavam alterações no nível da organização ou somente no nível dos indivíduos ou grupos (Burke, 2008; Cummings \& Worley, 2005; Van de Ven \& Poole, 2005; Weick \& Quinn, 1999).

Natureza da amostragem ou dos participantes

Avalia se é estudo de caso (poucos participantes de uma única organização), se houve amostragem (estudo de amostra, uma organização) e estudo de amostra com várias organizações.

Natureza da pesquisa empírica

No caso dos estudos empíricos, avalia se a abordagem de coleta e análise de dados foi qualitativa, quantitativa ou quali-quanti. 
Natureza do estudo

Origem dos dados

Procedimento de análise dos dados

Segmento da economia

Setor estudado

Tipo de instrumento de coleta de dados

Titulação do autor (p. ex., mestre, doutor)
Avalia se o estudo foi desenvolvido em uma pesquisa com desenho, relato de experiência, ensaio ou revisão de literatura.

Avalia se a pesquisa foi elaborada com dados primários (coletados pelo autor) ou secundários (coletados por terceiros).

Classifica a forma de análise dos dados utilizada: estatística inferencial ou descritiva ou análise de conteúdo ou do discurso.

Classifica o setor da economia da organização do estudo: primário, secundário ou terciário (serviços).

Avalia o setor de atuação das organizações estudadas (público, privado ou terceiro setor - ONG), aplicado a artigos de pesquisa empírica

Questionário/escala, entrevista, observação, investigação de documentos, testes/ prova ou outros.

Avalia a titulação dos autores do artigo. Artigos elaborados por mestres ou doutores.

Nota. *Categorias que se fundamentaram em autores específicos.

\section{RESULTADOS}

Para fins de organização da apresentação dos resultados foram adotados tópicos que descrevem inicialmente informações gerais sobre os artigos, como por exemplo, número de artigos por ano e origem dos autores. No segundo segmento estão descritos dados sobre o método utilizado nos artigos pesquisados, tais como abordagem metodológica e técnicas de coleta e análise de dados. Por fim, são descritas as informações sobre a caracterização das mudanças estudadas e os temas de pesquisa abordados.

\section{Informações gerais sobre os artigos}

De maneira geral, foram encontrados 7 artigos do ano 2000, 11 de 2001, 12 de 2002, 8 de 2003, 9 de 2004, 10 de 2005, 13 de 2006, 7 de 2007, 8 de 2008, 6 de 2009 e 6 de 2010. Há pequenas oscilações com decréscimos seguidos de períodos com crescimento constante, mas os resultados indicam publicação constante sobre o tema no período, conforme mostra a Figura 1.

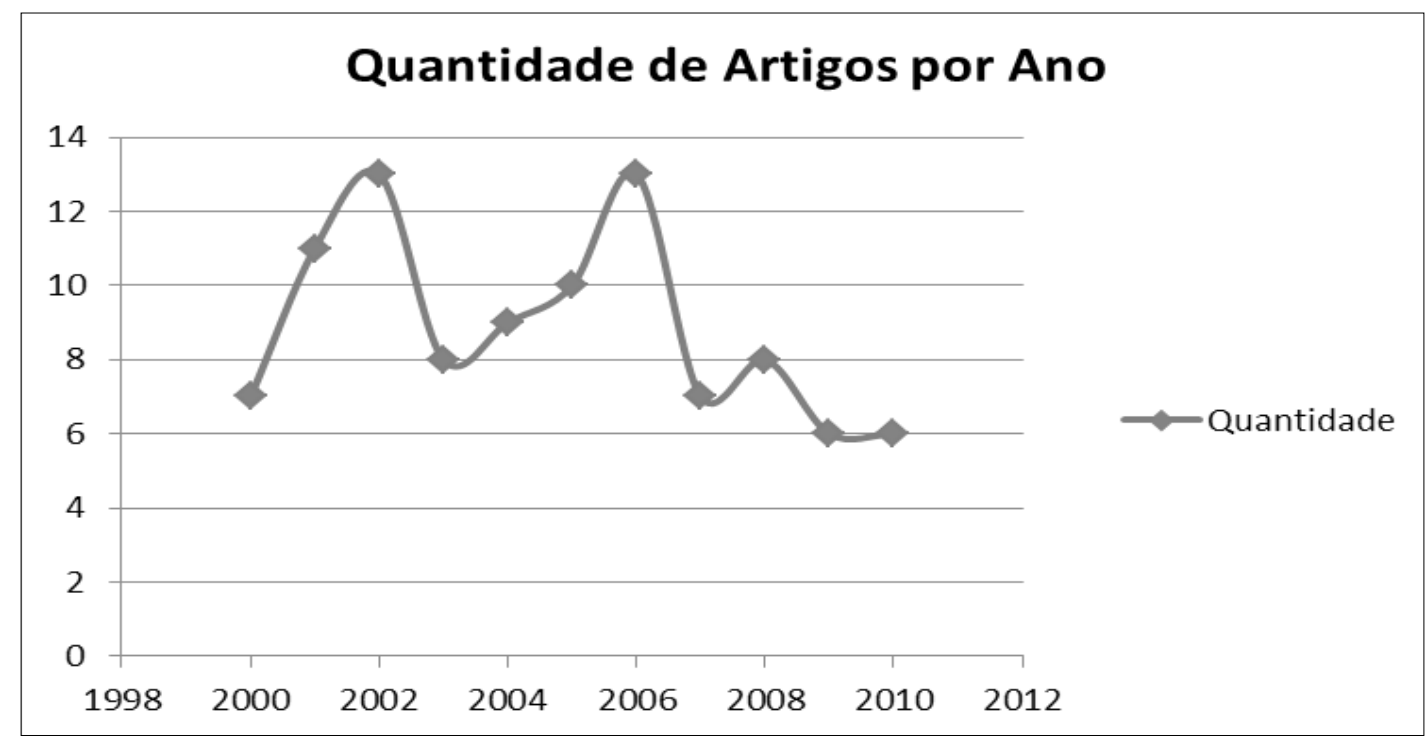

FIGURA 1. Distribuição dos artigos por ano

Muitas vezes a área de origem dos autores não está disponível em algumas revistas. Dos 97 artigos, apenas em 80 foi possível encontrar tal informação. Do total, 51 autores são da área da administração, 18 da psicologia, 3 das ciências sociais, 3 da área das ciências da saúde, 2 da informática, 2 da engenharia e 1 da educação. Para esse dado foi considerada a formação do primeiro autor. É possível observar uma evolução dos periódicos, uma vez que os artigos mais recentes já apresentam essa informação. Apenas em 38 artigos foi possível averiguar se as pesquisas possuíam vínculo com algum trabalho de conclusão de curso da pós-graduação. Destes, 13 eram originários de teses, 12 de dissertações e 13 de consultorias ou trabalhos de pesquisa específicos de professores.

Para investigar as informações sobre os segmentos da economia, a finalidade dos estudos e o tipo de organização estudada, foram considerados todos os artigos que apresentassem dados empíricos $(n=87 ; 9$ ensaios e 1 revisão de literatura foram excluídos). Assim, 57 artigos estudaram organizações do setor terciário, 12 eram 
estudos em organizações do setor secundário e 8 descreviam estudos e pesquisas em organizações do setor primário. Além destes, 3 estudaram organizações do setor primário e secundário; 4, organizações dos setores terciário e secundário; e 3, organizações dos três setores da economia. É importante destacar que somente 10 artigos traziam dados coletados em organizações que envolviam mais de um setor da economia (setores terciário e primário ou setores secundário e terciário).

Sobre a finalidade dos estudos, 11 artigos foram classificados como estudos exploratórios, 8 pretendiam gerar tecnologia para empregar nas organizações estudadas, 6 objetivavam gerar instrumentos de pesquisa para novos estudos e 62 tinham como meta gerar conhecimento. É importante ressaltar que os estudos poderiam apresentar outros objetivos, mas foi analisado apenas o que estava relatado como objetivo geral do artigo. Quanto ao tipo de organização estudada, 53 artigos eram sobre organizações privadas; 23, organizações públicas; 11 envolviam tanto organizações públicas quanto privadas; 9 eram artigos teóricos; e 1 tratava-se de revisão de literatura.

\section{Informações sobre o método de pesquisa}

Dos artigos encontrados, 73 apresentavam pesquisas com desenho metodológico, 14 envolviam relatos de experiência sobre mudança sem método sistematizado, 9 eram ensaios teóricos sobre as mudanças nas organizações sem qualquer dado empírico e um artigo trazia uma revisão de literatura sobre aspectos microorganizacionais das mudanças. Esse último manuscrito trazia como dados, as informações sobre artigos publicados. Portanto, para fins de classificação quanto ao método, apenas 73 artigos foram usados e os demais foram descartados.

Para fins de avaliação dos aspectos metodológicos, os 73 artigos foram classificados quanto ao desenho da pesquisa e utilizados para avaliar uma série de características metodológicas. E, quanto à natureza do desenho de pesquisa, 4 foram classificados como tendo um desenho quase-experimental, 63 foram classificados como levantamento de dados quantitativo ou qualitativo com recortes transversais e 6 apresentaram um desenho classificado como pesquisa-ação. Apenas 2 estudos, daqueles classificados como quase-experimentais, podem ser considerados como sendo de caráter longitudinal, uma vez que os autores coletaram os dados antes e após o processo de mudança com os mesmos sujeitos de pesquisa (Rossi et al., 1999).

$\mathrm{Na}$ maioria dos trabalhos (68 artigos), não há como comparar os diferentes momentos do processo de mudança. Nesses artigos, os trabalhos realizam avaliações post facto do que ocorreu a partir de informações de documentos ou percepções dos gerentes ou colaboradores. De maneira geral, esses artigos descreviam o que ocorreu durante o processo de mudanças por meio das histórias verbalizadas pelos sujeitos, o que retrata a construção social do processo de mudança.

A quantidade de estudos que adotou abordagem qualitativa na coleta de dados foi de 43; 22 apresentaram abordagem qualitativa e quantitativa simultaneamente; e 8 abordavam somente quantitativa. O mesmo ocorreu com estudos de caso (44 artigos), seguidos por uma amostra composta de sujeitos de somente uma organização (15), e, depois, uma amostra composta por sujeitos de várias organizações (14).

Ao analisar o tipo de procedimento de coleta, o procedimento de análise de dados e a fonte dos dados coletados (primários ou secundários), foram excluídos os artigos de natureza teórica e revisão de literatura. Os relatos de experiência foram incluídos para fins de verificação da origem e de método adotado para sistematização dos dados. Foi possível acrescentar os relatos de experiência também nesse tópico, pelo fato de todos apresentarem tais informações. A maioria dos estudos (57) utilizou mais de uma técnica de coleta de dados, sendo que a entrevista foi usada em 49 estudos. Em 16 estudos, a entrevista foi a única técnica de coleta de dados utilizada, o que demonstra certa ênfase em seu uso. Esses dados podem ser vistos na Tabela 4.

TABELA 4. Descrição dos métodos de pesquisa mais usados nos estudos

\begin{tabular}{lc}
\hline \multicolumn{1}{c}{ Instrumentos } & Frequência \\
Entrevista & 16 \\
Entrevista e questionário & 15 \\
Entrevistas e análise de documentos & 12 \\
Entrevista e observação livre & 10 \\
Questionário & 9 \\
Análise de documentos e de indicadores econômicos da empresa & 8 \\
Análise de documentos, entrevistas, observação livre & 7
\end{tabular}


Dos tipos de procedimentos de análise de dados, 42 apresentavam procedimento de análise de conteúdo ou do discurso, mais baseado na interpretação; 14 artigos continham análise de conteúdo sistemática com categorias; 11 apresentavam análises estatísticas descritivas; 11 apresentavam análises estatísticas descritivas e de conteúdo; 6 apresentam estatísticas inferenciais; e 3 apresentavam análises estatísticas descritivas, análises estatísticas inferenciais e análise de conteúdo. Quanto à origem dos dados, 63 artigos contaram com dados primários, coletados pelos autores dos trabalhos; 18 apresentavam dados primários e secundários; e 6 trabalharam com dados secundários.

\section{Dados sobre mudanças organizacionais e temas estudados}

Para avaliação das categorias referentes à caracterização das mudanças organizacionais, a classificação contava inicialmente com os 97 artigos avaliados. Contudo, 17 foram retirados por não se adequarem aos propósitos da classificação. Os artigos teóricos e a revisão de literatura oscilavam sobre as mudanças organizacionais e as mudanças nas organizações, abordando tanto intervenções com escopo, que envolvia todos os empregados, quanto intervenções mais focais, centradas em uma única área. Outras vezes abordavam efeitos tanto no nível macro de análise quanto no nível do indivíduo e, por isso, foram retirados da análise. Assim, dos 87 artigos restantes (extraídos os 9 teóricos e a revisão de literatura), 7 também foram retirados por não apresentarem informações que permitissem caracterizar o escopo das intervenções realizadas.

Para tratar de mudança, considerou-se como critério o fato de o artigo focalizar as alterações ocorridas nas organizações ou no comportamento das pessoas durante delimitado período de tempo. Alguns desses estudos fazem inferências sobre o processo de MO sem trazer informações sobre as intervenções realizadas, como um artigo que levantava atitudes em relação à $\mathrm{MO}$ em um contexto em que não havia mudanças nas organizações investigadas durante a pesquisa, embora existisse histórico de mudanças anteriores nas organizações (tais mudanças anteriores não foram descritas pelos autores).

A partir dessa reclassificação, apenas 80 artigos foram usados para análise das questões que se referem à MO ou mudança nas organizações. Destes, 21 tratavam de mudança nas organizações, e 59, de MO. Quanto à tipologia de mudanças, dos 80 artigos, 48 foram classificados como estudos sobre mudanças contínuas (p. ex., Dias, 2000), e 32, como estudos sobre mudanças epísodicas ou descontínuas (p. ex., Chaves, Santos, \& Morais, 2004).

Quanto à classificação dos artigos sobre envolver conteúdo, processo, contexto ou critério (resultados) de mudanças, dos 87 estudos (extraídos os artigos teóricos e a revisão de literatura), 18 abordavam tópicos relacionados ao contexto de mudanças, ou seja, envolviam fatores externos à organização que provocavam e possuíam efeito sobre seus resultados. Dos demais, 40 artigos abordavam conteúdos de MO (p. ex., Uribe, 2008), 20 estudaram o processo de mudança (p. ex., Caldas, 2000), e 15, os resultados das mudanças a partir de critérios comportamentais ou afetivos (p. ex., Blattmann, Borges, \& Bernardes, 2002). Nesse tópico, 6 artigos foram classificados em duas categorias por abordar dois aspectos. Essa classificação foi feita com base nos fenômenos abordados pelos artigos, e não com análise das intervenções apresentadas, portanto, foi possível utilizar todos os estudos.

Quanto ao fato de a pesquisa avaliar a percepção das pessoas sobre as mudanças, 21 artigos não investigaram tal fenômeno (p. ex., Oltramari \& Piccinini, 2006); 5 o investigaram, mas não encontraram mudanças percebidas pelos indivíduos; e os demais (54) investigaram tal fenômeno e concluíram que os membros das organizações perceberam que a instituição apresentou alterações ao longo do tempo (p. ex., Chaves et al., 2004; Martins \& Paz, 2000; Neiva \& Paz, 2007).

Considerando a profusão de conceitos e ideias apresentadas pelos artigos, foi realizada uma categorização específica em relação aos temas abordados. Nesse caso, foram analisados somente os artigos empíricos, e alguns foram classificados em duas ou mais categorias. Os estudos abordavam temas que variavam de planejamento 
estratégico, cultura organizacional e reações à mudança a reestruturação produtiva a partir de alterações nas organizações.

É importante ressaltar que tal análise foi bastante complexa na medida em que fenômenos similares foram analisados a partir de um arsenal diversificado de conceitos. Outra questão importante diz respeito à fundamentação teórica dos artigos. Não há um conjunto de teorias que fundamente a maioria dos artigos na área. Dos 97 estudos analisados, somente 44 utilizaram alguma teoria organizacional de suporte. Os que apresentavam algum referencial teórico eram 38, e 15 não faziam menção a um referencial teórico. Uma quantidade considerável (15 artigos) não faz menção a suporte teórico, apenas se ocupa em definir alguns conceitos utilizados, em um trabalho que se sustenta somente em dados empíricos.

Os temas que preponderaram nos estudos sobre MO são distribuídos em Estrutura, missão, estratégia e modelos de gestão organizacional; seguido por Efeitos das mudanças organizacionais: reaçôes emocionais, estresse, ansiedade, sofrimento elou adoecimento; Gestão da mudança organizacional e perfil dos gestores; e Aprendizagem no trabalho e nas organizações. Na categoria Estrutura, missão, estratégia e modelos de gestão organizacional, foram incluídos um conjunto de estudos que se dedica a avaliar se os programas idealizados pelo planejamento estratégico alcançam seus objetivos. Nesse conjunto, existem vários tipos de intervenções que procuram reestruturar a organização, reposicioná-la em relação ao mercado ou ambiente em que atua, criar novos produtos ou serviços e, ainda, realizar alterações na sua missão ou visão, como no estudo de Villas, Fonseca, Macedo-Soares e Diana (2006). Não foi identificado nenhum caso de alteração completa da missão, contudo, ressignificações ou ampliações estão presentes.

A segunda categoria, Efeitos das mudanças organizacionais: reações emocionais, estresse, ansiedade, sofrimento e/ou adoecimento, inclui todo o tipo de estudo que se preocupava com as reações emocionais dos empregados no contexto de MO. São estudos post facto interessados nessas emoções, suscitadas pelos programas de mudanças. Tais artigos dedicam-se a analisar temas sobre os chamados efeitos colaterais das mudanças ou efeitos que não eram seu principal objetivo (p. ex., Azevedo, Neto, \& Castilho Sá, 2002). Os estudos da terceira categoria, Gestão da mudança organizacional e perfil dos gestores, preocupam-se prioritariamente com o processo de MO. Esse tipo de estudo se dedica a descrever o processo de MO, especificando o que foi realizado na organização, e procura estabelecer prescrições sobre como deve ser a atuação gerencial durante as intervenções.

A quarta categoria envolve os estudos sobre Aprendizagem no trabalho e nas organizações, que objetivam especificar qual foi o grau de aprendizagem que as intervenções para a mudança proporcionaram aos indivíduos e à organização, como no estudo de Villardi e Leitão (2000). Trata de construtos como aprendizagem organizacional e outras formas de aprendizagem coletiva que são concebidos como resultados de mudanças organizacionais. Outros estudos procuram estabelecer relações entre eventos educativos e o processo de $\mathrm{MO}$ (p. ex., Ghedine, Testa, \& Freitas, 2006; Mourão \& Borges-Andrade, 2005). Há constantemente, nesses artigos, uma inferência de aprendizagem organizacional a partir de verificações da aprendizagem dos indivíduos e grupos, conforme mostra a Tabela 5 .

TABELA 5. Temas dos artigos analisados

\begin{tabular}{lc}
\multicolumn{1}{c}{ Temas } & N \\
\hline Estrutura, missão, estratégia e modelos de gestão organizacional & 16 \\
Gestão da mudança organizacional, perfil e ação dos gestores & 13 \\
Efeitos das mudanças organizacionais: reações emocionais, estresse, ansiedade, sofrimento e/ou adoecimento & 11 \\
Aprendizagem no trabalho e nas organizações decorrentes das mudanças & 9 \\
Mudanças na cultura organizacional de fusões, aquisições, privatizações e outras intervenções & 8 \\
Mudanças na gestão de pessoas e efeitos nas relações de trabalho & 6 \\
Atitudes, cognições ou crenças em relação à mudança organizacional & 6 \\
Resistência à mudança organizacional & 5 \\
Implementação da tecnologia de informação/comunicação & 4 \\
Efeitos de programas organizacionais nos clientes ou usuários dos serviços - relação cliente/organização & 4 \\
Impactos das mudanças nas competências, desempenho e produtividade no trabalho & 4 \\
Efeitos dos programas de downsizing & 3 \\
Avaliação dos efeitos dos programas de qualidade & 3 \\
Alianças entre organizações no processo de MO e competição por recursos & 3 \\
Relações de poder, relações entre grupos e redes sociais na mudança & 2
\end{tabular}


Outros temas que merecem destaque são os de mudança cultural nas organizações e os de resistência à mudança organizacional e atitudes, cognições ou crenças em relação à MO. Nos artigos, esses temas aparecem associados porque não há muita diferenciação entre esses tópicos. Eles abordam as reações emocionais posteriores à implantação das intervenções, as resistências à mudança e as atitudes, crenças ou outras cognições que envolvem o processo de mudança. Existem estudos teóricos na literatura que se preocupam com a definição e a diferenciação do fenômeno da resistência com algumas confusões entre os conceitos de emoções, cognições, afetos e comportamentos em relação à MO (Hernandez \& Caldas, 2001; Silva \& Vergara, 2003).

O tema mudança na cultura organizacional também aparece com muitas leituras diferenciadas. Algumas inferências sobre as mudanças que aconteceram na organização servem para atribuir ao episódio um processo de mudança cultural (como no estudo de Rossini, Crubellate, \& Mendes, 2001). Há também uma tendência em responsabilizar a cultura pelas dificuldades de concretizar as mudanças pretendidas.

\section{DISCUSSÃO}

De maneira geral, os objetivos do artigo foram alcançados e foi possível concluir algumas tendências metodológicas, bem como algumas deficiências ainda presentes no que foi produzido neste período. A produção reflete de maneira geral a ocorrência de vários eventos de mudança organizacional nos últimos dez anos nas organizações brasileiras. Trata-se de uma resposta dos pesquisadores às demandas dos profissionais que são envolvidos em muitas intervenções cujo objetivo é criar uma nova proposta de funcionamento que atenda às exigências do mercado (Weick \& Quinn, 1999).

\section{Informações gerais sobre os artigos}

Os resultados apontam para uma publicação constante, o que demonstra o interesse dos pesquisadores pelo fenômeno, apesar da pulverização da literatura, panorama que está em conformidade com as postulações de autores internacionais (Weick \& Quinn, 1999). Várias áreas, inclusive a da saúde, se preocupam com o fenômeno da $\mathrm{MO}$, o que reforça seu caráter interdisciplinar. Muitos trabalhos são vinculados a consultorias, embora a maioria possua origem em trabalhos de dissertações, teses ou pesquisas acadêmicas. Esse dado demonstra a preocupação acadêmica em compreender a sistemática do fenômeno.

Muitos estudos envolvem organizações privadas e vinculam-se a organizações do setor terciário da economia: o de serviços. Talvez esse dado esteja relacionado a uma preponderância de eventos de $\mathrm{MO}$ nessas organizações. Há necessidade de estudos que avaliem os processos de $\mathrm{MO}$ em organizações públicas, que constantemente sofrem intervenções e alterações de gestão.

\section{Informações sobre o método de pesquisa}

No que diz respeito às características metodológicas dos estudos, a maioria se trata de pesquisas qualitativas, o que revela uma priorização de uma das tendências apontadas por Van de Ven e Poole (2005). Estudos qualitativos usados isoladamente são característicos de problemas de pesquisa exploratórios em sua natureza e de áreas de estudos que possuem fundamentação teórica pulverizada e incipiente (Creswell, 2007). Essa afirmativa é bastante questionável, mas é importante atentar para a preponderância de estudos que se centram na construção do significado.

Outro problema encontrado nos artigos é a grande presença dos estudos de caso que têm a característica de pesquisar o fenômeno em profundidade, mas limitado poder de generalização (Creswell, 2007). Há abordagens epistemológicas que questionam o quanto se pode concluir padrões de conhecimento na área das ciências humanas, mas a possibilidade das conclusões serem estendidas para outras organizações e grupos deve ser minimamente analisada nesse tema da MO. Precisa-se investigar existência de leis gerais e padrões nos fenômenos para que seja possível afirmar categoricamente que eles não existem.

Dos artigos analisados, 42 indicaram um procedimento genérico de análise de conteúdo que, muitas vezes, não apresenta categorias extraídas das verbalizações. Nesse caso, há necessidade de maior sistematização das análises, com indicação de como os procedimentos foram realizados. Poucos estudos se dedicam a análises de conteúdo sistematizadas ou análises estatísticas inferenciais, na medida em que aqueles que utilizaram tais 
procedimentos se concentraram apenas em apresentar dados descritivos. Tal informação corrobora a preponderância de pesquisas descritivas nesse campo, o que retrata o caráter inicial da área.

Apesar da natureza predominantemente exploratória dos estudos, 9 artigos são de natureza teórica, constituindo ensaios sobre o fenômeno da MO. Tais artigos discutiam definições e propostas para análise do fenômeno, embora muitos tenham trazido exemplos não sistematizados de casos que respaldavam a discussão. Apenas dois estudos não faziam menção a qualquer evento de $\mathrm{MO}$ que pudesse respaldar a teorização. De acordo com Creswell (2007), a proposição de teorias em uma área que não possui um corpo de pesquisas sistematizado e consolidado pode tornar-se infrutífera, com risco de as formulações não fornecerem um respaldo para problemas de natureza empírica. É importante distinguir questões empíricas de questões conceituais e empreender esforços na solução de ambos os problemas.

Ainda quanto ao aspecto metodológico, pode-se verificar a grande presença de estudos sem qualquer fundamentação teórica (15 artigos), o que caracteriza uma produção científica que limita o avanço do conhecimento na área. No entanto, o quadro apresenta aspectos positivos na medida em que 17 artigos atendem às formulações de Pettigrew (1995) e Woodman (1989), que enfatizam a necessidade de abordagens com uso de vários métodos de coleta e análise de dados, com integração e triangulação de informações de naturezas qualitativa e quantitativa. Principalmente pela complexidade da mudança organizacional, uma abordagem combinada de vários procedimentos metodológicos auxilia na compreensão do fenômeno. Tal realidade ainda reforça o caráter exploratório desse campo do conhecimento em virtude de essas características metodológicas estarem associadas prioritariamente a estudos descritivos que são limitados para estabelecer relações entre variáveis e testar teorias.

\section{Dados sobre as mudanças organizacionais e temas estudados}

A maioria dos artigos investigou intervenções de grande escopo (59 foram classificados como MO), o que indica alguma propriedade no uso do termo. Existem muitos artigos que tratam de intervenções de menor amplitude e intensidade, que não envolvem toda a organização ou os aspectos relacionados a mudanças mais radicais ou descontínuas, como estratégia, ambiente externo, cultura e missão (Burke, 2008; Burke \& Litwin, 1992). Algumas vezes, atribui-se possibilidade de mudança cultural a programas mais restritos, como os de qualidade e a gestão por competência. Nesse caso, há necessidade de maior investigação das ações realizadas e seus objetivos alcançados para verificar em que medida elas realmente são capazes de provocar MO. Contudo, a delimitação dos programas visando avaliar seus efeitos ainda é precária nos estudos. A maioria também trata de várias ações realizadas ao longo do tempo e não consegue estabelecer comparações entre o que foi realizado e o que realmente aconteceu. Há um conjunto diversificado de atividades realizadas que são classificadas como desencadeadoras de mudanças.

Ressalta-se a necessidade de caracterizar melhor as intervenções e delimitar seu escopo em termos de impactos possíveis e alcançados, discriminando e separando algumas especulações que não têm grande respaldo empírico, como atribuir uma mudança cultural a um programa de qualidade ou de gestão por competências. Há intervenções muito diferenciadas que são tratadas da mesma forma pela literatura nacional, o que não é muito diferente do que tem sido apontado pelas revisões de literatura internacional (Pettigrew et al., 2001). Modelos teóricos, um corpo teórico comum e um paradigma dominante ainda não foram desenvolvidos, e muitas revisões da literatura internacional apontam essa profusão de microteorias e dificuldade de avançar em um corpo teórico mais estruturado sustentado por respaldo empírico (Armenakis \& Bedeian, 1999; Pettigrew et al., 2001; Weick \& Quinn, 1999). Contudo, também ressaltam a complexidade do fenômeno e a dificuldade de construir conhecimento nesse contexto.

Verifica-se também que as pesquisas analisadas utilizaram diferentes tipos de intervenções (inovações tecnológicas, alterações estruturais, programas de qualidade e programas educativos) e isso impossibilita, mais uma vez, a comparação e generalização dos resultados. Tais intervenções e contextos de mudança podem ser percebidos pelos indivíduos de maneiras distintas, o que requer uma análise das relações entre o que foi realizado, o que foi percebido como conteúdo de mudança e as reações dos empregados ao processo. A maioria dos artigos preocupa-se com a construção do significado da mudança, o que reforça apenas uma tendência apresentada por Van de Ven e Poole (2005).

Woodman (1989) aponta a diferença entre a percepção dos indivíduos sobre a mudança e os indicadores de produtividade utilizados nos estudos, questão investigada nos artigos avaliados na literatura nacional. Parece 
não haver uma tendência para a investigação da percepção como um dos indicadores de MO. Muitos estudos se concentram somente em indicadores objetivos da organização ou usam relatórios e documentos institucionais como indicador de mudanças. $\mathrm{O}$ uso de documentos da organização pode ser equivocado na medida em que muitos deles são elaborados a partir das percepções de alguns de seus membros, além da inexistência de controle sobre as informações apresentadas nos documentos.

Pettigrew (1995) e Pettigrew et al. (2001) ressaltam a necessidade de uso de múltiplas fontes de dados para análise do processo em virtude da falta de controle sobre as informações. Ford e Ford (1994), Neiva (2004) e Woodman (1989) ressaltam o papel da percepção no processo de definição do fenômeno da MO. Há uma atribuição das pessoas sobre o alcance dos objetivos pelas intervenções realizadas. Além disso, fenômenos como cultura, aprendizagem, missão e visão compartilhadas são acessíveis somente por meio da investigação da percepção dos indivíduos nas organizações. Outro fato relacionado a isso é a necessidade de reconhecimento de que o envolvimento do sujeito no processo de mudança, sua participação e a oportunidade de expressão são fundamentais para a que mudança seja bem-sucedida (Coyle-Shapiro, 1999; Damanpour, 1992). Uma mudança radical implica em alteração dos modelos de gestão, e a percepção é um indicador fundamental para reconhecimento de que o processo foi bem-sucedido. Portanto, sugere-se que os próximos estudos atentem para a avaliação de indicadores de produtividade e os associem à percepção dos indivíduos.

Ainda é preocupante que artigos não apresentem informações mínimas sobre as intervenções realizadas. Neiva (2003) discute a possibilidade de estudo da MO sem mensurar qualquer aspecto relacionado à intervenção, apenas especificando características organizacionais indicadas por alguma teoria, como é o caso do estudo de Martins e Paz (2000). As informações sobre as intervenções são fundamentais para discussão ou inferências sobre as relações de associação ou relações causais entre o que foi realizado e as alterações detectadas. Quando se foca apenas em alguma característica organizacional, de grupos ou de membros, há uma tendência a tentar descrever o desenvolvimento da organização sem vínculo com as intervenções realizadas, o que pode ser feito, mas não permite inferências sobre efeitos de intervenções, como ocorre em muitos estudos.

Existe uma distribuição dos estudos nacionais sobre MO nos temas de pesquisa postulados por Armenakis e Bedeian (1999). Embora os estudos sobre conteúdo de mudança sejam prioritários, há investimentos nos estudos de processo e critério (resultados colaterais da mudança) e uma atenção aos estudos de contexto. O interesse pelos estudos de critério, que envolvem reações emocionais à mudança, ao estresse, ao adoecimento e ao bem-estar pessoal, está em consonância com as indicações da literatura internacional que apontam a necessidade de estudos desses fenômenos e da relação destes com as intervenções realizadas (Armenakis \& Bedeian, 1999). Tal realidade tem um respaldo prático na medida em que auxilia o planejamento de processos de mudanças que causem menor impacto negativo na vida dos membros organizacionais.

A questão das reações e resistências a mudanças organizacionais é constantemente levantada em um contingente de artigos sobre o tema. A categorização de fenômenos e temas abordados nos estudos sobre MO revela grande interesse dos autores sobre tais aspectos. Nesse tópico, é importante ressaltar a confusão entre fenômenos que envolvem o nível individual que são definidos como resistência a mudanças. As reações emocionais dizem respeito às emoções que os empregados desenvolvem após a implantação do programa de mudança em decorrência do contexto que se estabelece e das características das intervenções.

As resistências, por sua vez, se referem a respostas comportamentais (ações) que os colaboradores realizam com o objetivo de se oporem ao programa de mudanças por suas características fundamentais. E o tema atitudes, crenças ou cognições sobre a mudança envolvem o que os empregados pensam antes e/ou depois do programa de mudanças que será implantado, como no estudo de Chaves et al. (2004). No relato do autor, esses conceitos, na maioria dos casos, não são diferenciados. Ajzen e Fisbein (1980) fazem uma distinção clara entre esses aspectos, explicitando a relação entre as cognições e afetos e o comportamento posterior.

$\mathrm{Na}$ análise da resistência às mudanças organizacionais, é importante ressaltar a contribuição de autores como Hernandez e Caldas (2001) e Silva e Vergara (2003) ao questionarem o uso do conceito de resistência para classificar comportamentos manifestos por empregados durante os processos de mudanças organizacionais. Muitos comportamentos classificados nessa categoria podem revelar aspectos do processo de mudanças, como falta de informação, de planejamento ou suporte às ações desenvolvidas por parte dos coordenadores das intervenções.

Outra contribuição importante diz respeito à proposição teórica de um modelo de etapas da $\mathrm{MO}$ e da gestão estratégica, associando esta e o planejamento a gestão da mudança (Estrada \&Almeida, 2007). Existem poucos 
modelos de processo de mudanças na literatura sobre o tema. De acordo com Armenakis e Bedeian (1999), o modelo de etapas de mudanças mais considerado na literatura é o de Kotter (1995). Estrada e Almeida (2007) associam as etapas propostas por Kotter (1995) às etapas da gestão estratégica teorizadas por eles.

Uma conclusão possível diz respeito à pouca diversificação e sistematização dos procedimentos de coleta e análise de dados. Em virtude de a $\mathrm{MO}$ se caracterizar pela complexidade, a abordagem multimétodo pode colaborar bastante (Neiva, 2003; Pettigrew, 1995; Pettigrew et al., 2001). A abordagem multimétodo se caracteriza pelo uso de vários e diferenciados procedimentos de coleta e análise de dados para investigar o mesmo fenômeno, pois o uso de vários métodos tende a aumentar a validade interna dos estudos. Os resultados dessa revisão mostraram uma concentração grande em uma única técnica e pouca triangulação das informações levantadas.

O retrato a partir dessa revisão de literatura aponta para o possível crescimento da área, mas ainda reflete pouca maturidade dos estudos para discussões aprofundadas sobre o fenômeno. Essa pouca maturidade evidencia-se na grande ênfase da abordagem qualitativa, de caráter interpretativo, e uma presença considerável de estudos de casos e estudos sem fundamentação teórica. Van de Ven e Poole (2005) afirmam que essas tendências refletem concepções diferenciadas de organização, mas ressaltam a necessidade de combinar as duas abordagens para a compreensão do fenômeno da MO. Essa realidade é facilmente compreensível, dada a complexidade da MO. O grande desafio das pesquisas é avançar no sentido de maior sistematização do conhecimento, com estabelecimento de indicadores e instrumentos para mapeamento do contexto; e o estabelecimento de relações entre o que foi objetivado pela intervenção e as alterações identificadas ou percebidas.

Outro avanço seria no sentido de especificar de maneira sistematizada as características do contexto e as reações dos indivíduos em função dos diversos processos de mudança por quais passam as organizações, que são entidades estruturadas e processos sociais contínuos quando vistas sob prismas diferenciados (Tsoukas \& Chia, 2002). Portanto, priorizar uma única concepção de organização e uma única abordagem metodológica pode limitar a construção de conhecimento em um objeto reconhecidamente complexo.

\section{REFERÊNCIAS}

Ajzen, I., \& Fisbein, M. (1980). Understanding attitudes and predicting social behavior. Englewood Cliffs: Prentice-Hal.

Armenakis, A. A., \& Bedeian, A. G. (1999). Organizational change: A review of theory and research in the 1990s. Journal of Management, 25(3), 293-315.

Azevedo, C. S., Neto, F. C. B., \& Castilho Sá, M. (2002). Indivíduo e mudança nas organizações de saúde: Contribuições da psicossociologia. Cadernos de Saúde Pública, 18(1), 235-247.

Blattmann, U., Borges, I., \& Bernardes, L. L. R. (2002). Mudança organizacional e o local de trabalho: Reflexões. Revista ACB: Biblioteconomia em Santa Catarina, 7(1/2), 240-250.

Borges-Andrade, J. E., Coelho Jr., F. A., \& Queiroga, F. (2006). Pesquisa sobre micro comportamento organizacional no Brasil: O estado da arte. Anais eletrônicos do I/ Congresso Brasileiro de Psicologia Organizacional e do Trabalho. Brasília: Universidade de Brasília.

Burke, W. W. (2008). Organization change: Theory and practice (2 ed.). Londres: SAGE Publications.

Burke, W. W., \& Litwin, G. H. (1992). A causal model of organizational performance and change. Journal of Management, 18(3), 523-545.

Caldas, M. P. (2000). Enxugamento de Pessoal no Brasil: Podem-se atenuar seus efeitos em empresa e indivíduo? Revista de Administração de Empresas, 40(1), 29-41.

Chaves, A. M., Santos, M. V., \& Morais, J. H. M. (2004). Representação social de mudança organizacional: Estudo de caso numa empresa petroquímica de Salvador. Revista Psicologia: Organizações e Trabalho, 4(2), 63-84.

Coyle-Shapiro, J. A. (1999). Employee participation and assessment of an organizational change intervention: A three-wave study of total quality management. The Journal of Applied Behavioral Science, 35(4), 439-456.

Creswell, J. W. (2007). Projeto de pesquisa: Métodos qualitativos, quantitativos e misto (2 ed.). Porto Alegre: Artmed.

Cummings, T. G., \& Worley, C. G. (2005). Organization development and change (8 ed.). Nova York: Thompson.

Damanpour, F. (1992). Organizational innovation: A meta-analysis of effects of determinants and moderators. Academy of Management Journal, 34(3), 555-590. 
Dias, D. S. (2000). Motivação e resistência ao uso da tecnologia da informação. Revista de Administração Contemporânea, 4(2), 51-66.

Estrada, R. J. S., \& Almeida, M. I. R. (2007). A eficiência e a eficácia da gestão estratégica: Do planejamento estratégico a mudança organizacional. Revista de Ciências da Administração, 9(19), 147-178.

Ford, J. D., \& Ford, L. W. (1994). Logics of identity, contradiction and attraction in change. Academy of Management Review, 19(4), 756-785.

Ghedine, T., Testa, M. G., \& Freitas, H. M. R. (2006). Compreendendo as iniciativas de educação a distância via internet: Estudo de caso em duas grandes empresas no Brasil. Revista de Administração Pública - RAP, 40(3), 427-455.

Harzem, P., \& Miles, T. R. (1978). Conceptual issues in operant psychology. Nova York: John Wiley \& Sons.

Hernandez, J. M., \& Caldas, M. P. (2001). Resistência à mudança: Uma revisão crítica. Revista de Administração de Empresas, 41(2), 31-45.

Kotter, J. P. (1995). Leading change: Why transformation efforts fail. Harvard Business Review, 73(2), 59-67.

Lima, S. M. V., \& Bressan, C. L. (2003). Mudança organizacional: Uma introdução. In S. M. V. Lima (Ed.), Mudança Organizacional - Teoria e gestão (pp. 17-63). Rio de Janeiro: Editora FGV.

Martins, J. L. T. P., \& Paz, M. G. T. (2000). Poder e comprometimento em tempo de mudança organizacional: Estudo de caso de uma empresa pública de serviços de informática. Revista de Administração, 35(4), 61-71.

Mourão, L., \& Borges-Andrade, J. E. (2005). Avaliação de programas públicos de treinamento: Um estudo sobre o impacto no trabalho e na geração de emprego. Organizações \& Sociedade, 12(33), 13-38.

Neiva, E. R. (2003). Metodologia para avaliação da mudança organizacional. In S. M. Lima (Org.), Mudança organizacional: Teoria e gestão (pp. 191-215). São Paulo: Editora FGV.

Neiva, E. R. (2004). Percepção da mudança organizacional: O papel das atitudes e das características organizacionais (Tese de doutorado não publicada). Universidade de Brasília, Brasília.

Neiva, E. R., \& Paz, M. G. T. (2007). Percepção de mudança organizacional: Um estudo em uma organização pública brasileira. Revista de Administração Contemporânea, 11(1), 31-52.

Oltramari, A. P., \& Piccinini, V. C. (2006). Reestruturação produtiva e formas de flexibilização do trabalho. Organizações \& Sociedade, 13(36), 85-106.

Pettigrew, A. (1995). Longitudinal field of research on change: Theory and practice. In G. P. Huber \& A. H. Van de Ven (Orgs.), Longitudinal field research methods: Studying processes of organizational change (pp. 91-125). Londres: Sage Publications.

Pettigrew, A., Woodman, R., \& Cameron, K. S. (2001). Studying organizational change and development: Challenges for future research. Academy of Management Journal, 44(4), 697-713.

Rossi, P. H., Freeman, H. E., \& Lipsey, M. W. (1999). Evaluation: A systematic approach (6 ed.). Londres: Sage Publications.

Rossini, A. J., Crubellate, J. M., \& Mendes, A. A. (2001). Reação cultural à aquisição: Estudo do caso Santander/Noroeste. Revista de Administração Contemporânea, 5(1), 145-164.

Silva, J. R. G., \& Vergara, S. C. (2003). Sentimentos, subjetividade e supostas resistências à mudança organizacional. Revista de Administração de Empresas, 43(3), 10-21.

Tsoukas, H., \& Chia, R. (2002). On organizational becoming: Rethinking organizational change, Organization Science, 13(5), 567599.

Van de Ven, A., \& Poole, M. S. (1995). Explaining development and change in organizations. The Academy of Management Review, 20(3), 510-540.

Van de Ven, A., \& Poole, M. S. (2005). Alternative approaches for studying organizational change. Organization Studies, 26(9), 1377-1404.

Villardi, B. Q., \& Leitão, S. P. (2000). O conceito de organizações de aprendizagem e seu potencial para mudanças transformadoras nas organizações empresariais. Implicações para a teoria e a prática da Administração. Revista Brasileira de Administração Pública, 34(3), 53-70.

Villas, M., Fonseca, M., Macedo-Soares, T. D. L., \& Diana, V. A. (2006). Assegurando o alinhamento estratégico da tecnologia de informação e comunicação: $\mathrm{O}$ caso das unidades de refino da Petrobrás. Revista de Administração Contemporânea, 4O(1), 22-32.

Weick, K. E., \& Quinn, R. E. (1999). Organizational change and development. Annual Review of Psychology, 50, 361-386. 
Woodman, R. (1989). Evaluation research on organizational change: Arguments for a combined paradigm approach. Research in Organizational Change and Development, 3, 161-180.

Uribe, F. J. (2008). Reflexões sobre a subjetividade na gestão a partir do paradigma da organização que aprende. Ciência \& Saúde Coletiva, 6(1), 208-219. 